\section{Funk, Casimir}

\author{
A. M. Gressner ${ }^{1}$ und O. A. Gressner ${ }^{2}$ \\ ${ }^{1}$ Labor Dr. Wisplinghoff Berlin, Berlin, Deutschland \\ ${ }^{2}$ Labor Dr. Wisplinghoff Köln, Köln, Deutschland
}

Lebensdaten US-amerikanischer Biochemiker polnischer Herkunft, geboren am 23. Februar 1884 in Warschau, gestorben am 20. November 1976 in New York City.

Verdienste Casimir Funk erhielt seine schulische Bildung in Warschau und studierte Biologie und Chemie an den Universitäten Genf und Berlin. Nach der Promotion zum Ph.D. 1904 arbeitete Funk am Pasteur-Institut über organische Basen, Aminosäuren/Proteine und Zucker. Im Jahr 1906 wechselte er nach Berlin, wo er u. a. mit Emil Fischer und Emil Abderhalden Experimente zum Proteinstoffwechsel durchführte. Diese Studien, die er 1910 am Lister-Institut in London fortsetzte, lenkten seine Forschung auf die Klärung der Ätiologie und Pathogenese der Beriberi-Krankheit, die durch den Verzehr von poliertem Reis im Orient prävalent war. Er fand heraus, dass spezifische organische Komponenten in den Nahrungsmitteln essenziell für die Prävention und nachfolgende Therapie von Beriberi, Rachitis und Skorbut sind, für die er die kollektive Bezeichnung Vitamine (,vitality amines") um 1912 einführte. 1915 setzte Funk seine Forschung in den USA fort und wurde 1920 US-Bürger. Von 1920-1939 arbeitete Funk in Polen, kehrt jedoch kriegsbedingt 1939 in die USA (New York) zurück, um in der U.S. Vitamin Corporation bis $1963 \mathrm{zu}$ arbeiten. Funks Entdeckung der Vitamine wies deren präventive und kurative Wirkung für Erkrankungen wie perniziöse Anämie, Beriberi, Osteomalazie, Pellagra, Rachitis, Skorbut und Sprue nach.

\section{Literatur}

Funk C (1924) Die Vitamine, ihre Bedeutung für die Physiologie und Pathologie, 3. Aufl. J.F. Bergmann Verlag, München 